\title{
Overexpression of $\mathrm{HOXB7}$ homeobox gene in oral cancer induces cellular proliferation and is associated with poor prognosis
}

\author{
MARIA FERNANDA DE SOUZA SETUBAL DESTRO ${ }^{1 *}$, CAROLINA CAVALCANTI BITU $^{2 *}$, \\ KARINA G. ZECCHIN ${ }^{1,2}$, EDGARD GRANER ${ }^{1}$, MARCIO A. LOPES ${ }^{1}$, \\ LUIS PAULO KOWALSKI ${ }^{3}$ and RICARDO D. COLETTA ${ }^{1}$ \\ ${ }^{1}$ Department of Oral Diagnosis, School of Dentistry, State University of Campinas, Piracicaba; \\ ${ }^{2}$ Department of Clinical Pathology, School of Medicine, State University of Campinas, Campinas; \\ ${ }^{3}$ Department of Head and Neck Surgery and Otorhinolaryngology, A.C. Camargo Cancer Hospital, São Paulo, Brazil
}

Received July 7, 2009; Accepted September 3, 2009

DOI: 10.3892/ijo_00000485

\begin{abstract}
A growing body of evidence has confirmed the involvement of dysregulated expression of HOX genes in cancer. HOX genes are a family of 39 transcription factors, divided in 4 clusters (HOXA to HOXD), that during normal development regulate cell proliferation and specific cell fate. In the present study it was investigated whether genes of the HOXB cluster play a role in oral cancer. We showed that most of the genes in the HOXB network are inactive in oral tissues, with exception of HOXB2, HOXB7 and HOXB13. Expression of HOXB7 was significantly higher in oral squamous cell carcinomas (OSCC) compared to normal oral mucosas. We further demonstrated that HOXB7 overexpression in HaCAT human epithelial cell line promoted proliferation, whereas downregulation of HOXB7 endogenous levels in human oral carcinoma cells (SCC9 cells) decreased proliferation. In OSCCs, expression of HOXB7 and Ki67, a marker of proliferation, correlate strongly with each other $(\mathrm{rs}=0.79, \mathrm{p}<0.006)$. High immunohistochemical expression of HOXB7 was correlated with T stage $(\mathrm{p}=0.06), \mathrm{N}$ stage $(\mathrm{p}=0.07)$, disease stage $(\mathrm{p}=0.09)$ and Ki67 expression $(\mathrm{p}=0.01)$, and patients with tumors showing high number of HOXB7-positive cells had shorter overall survival $(\mathrm{p}=0.08)$ and shorter disease-free survival after treatment $(\mathrm{p}=0.10)$ compared with patients with tumors exhibiting low amount of HOXB7-positive cells. Our data suggest that HOXB7 may contribute to oral carcinogenesis by increasing tumor cell proliferation, and imply that HOXB7 may be an important determinant of OSCC patient prognosis.
\end{abstract}

Correspondence to: Dr Ricardo D. Coletta, Department of Oral Diagnosis, School of Dentistry, State University of Campinas, Piracicaba, São Paulo, Brazil

E-mail: coletta@fop.unicamp.br

*Contributed equally

Key words: oral cancer, homeobox genes, HOX, HOXB7, proliferation, clinicopathological correlation, survival

\section{Introduction}

HOX genes belong to the superfamily of homeobox gene that encodes for transcription factors with important roles in development (1). Homeoproteins control diverse cellular processes by regulating the expression of many downstream target genes. Thus, it is typical for an individual homeoprotein to confer pleiotropic effects on cell behavior, including alterations in proliferation, survival, migration, and invasion (2-6). In humans 39 HOX genes have been identified and they are organized into 4 paralogous clusters (HOXA, HOXB, HOXC and HOXD) on autosomal chromosomes (3-7). Genes of the HOX family are highly expressed in leukemias, melanomas and in breast, ovarian, cervical, esophageal and prostate cancers (8-14). In addition, aberrant expression of HOX genes was observed in oral squamous cell carcinomas (OSCC) (15). However, their relationships with the neoplastic phenotype remain unclear.

During normal development, HOXB7 stimulates the proliferation and survival of progenitor cells, and when expressed out of context, HOXB7 can aberrantly promote proliferation, contributing to tumorigenesis (16-18). HOXB7 has been shown to interact with proteins related to the DNA repair machinery, and its overexpression promotes transformation of human mammary epithelial cells (19). Although HOXB7 expression has been observed in OSCCs (15), its role in human oral cancers has not been reported. In this study, we analyzed the expression of HOXB genes in normal oral mucosas and OSCCs, and investigated the effect of HOXB7 on stimulation of proliferation and survival of OSCC cells. Additionally, we have analyzed the prognostic significance of HOXB7 immunohistochemical expression for OSCC patients.

\section{Material and methods}

Samples and clinicopathological data. For the initial expression screening of HOXB members by duplex reverse transcriptasepolymerase chain reaction (RT-PCR), this study analyzed fresh samples from normal oral mucosa obtained from 10 patients without history of exposure to risk factors related to 
OSCC such as smoking habit and alcohol consumption, and 14 pairs of fresh samples, each pair from the same patient, of OSCC and adjacent normal oral mucosa. Fresh samples were divided into two parts: one was fixed in formalin and embedded in paraffin for hematoxylin and eosin staining or immunohistochemistry, while the other was snap-frozen in liquid nitrogen.

To determine the clinicopathological correlation of the HOXB7 expression, immunohistochemistry was performed in primary OSCCs of 35 patients diagnosed and treated at the Department of Head and Neck Surgery and Otorhinolaryngology, A.C. Camargo Cancer Hospital, São Paulo, Brazil. The OSCC patients, 33 of which were males, ranged in age between 43 and 89 years, with a mean of $60.7 \pm 11.9$ years. History of alcohol consumption was recorded in 26 $(74.3 \%)$ and tobacco smoking in $29(82.9 \%)$ patients. The site of primary tumor was tongue $(n=14)$, floor of mouth $(n=7)$, palate $(n=7)$, buccal mucosa $(n=5)$, and gingiva $(n=2)$. The tumors were staged according to the International Union Against Cancer (TNM stage) as follows: stage I (4 patients), stage II (8 patients), stage III (9 patients), and stage IV (14 patients). All patients were staged as M0 at the time of diagnosis. Tumors were classified for their pattern of cellular differentiation as previously described by Anneroth et al (20). After treatment, patients were followed up monthly and disease recurrence was histologically confirmed.

Informed consent was obtained from each patient and the study was carried out with approval of the Human Research Ethics Committee of the School of Dentistry, State University of Campinas.

Cell culture and plasmids. The human OSCC cell line SCC9 was obtained from American Type Culture Collection (ATCC, Manassas, VA, USA), and cultured as recommended in 1:1 mixture of Dulbecco's modified Eagle's medium (DMEM) and Ham's F12 medium (DMEM/F12; Invitrogen, Carlsbad, CA, USA) supplemented with $10 \%$ fetal bovine serum (FBS), $400 \mathrm{ng} / \mathrm{ml}$ hydrocortisone (Sigma-Aldrich, St. Louis, MO, USA), $100 \mu \mathrm{g} / \mathrm{ml}$ penicillin, and $100 \mu \mathrm{g} / \mathrm{ml}$ streptomycin at $37^{\circ} \mathrm{C}$ in a humidified atmosphere of $5 \% \mathrm{CO}_{2}$. The HaCAT epithelial cell line was kindly provided by Dr Andre Luis Vettore (Ludwig Institute for Cancer Research, São Paulo Branch), and was maintained in DMEM containing $10 \%$ FBS and antibiotics at $37^{\circ} \mathrm{C}$ in a $5 \% \mathrm{CO}_{2}$ air atmosphere. HaCAT-HOXB7 stable cell lines were generated as previously described (21) using the HOXB7-pcDNA3 plasmid (22). Control cells for HOXB7 transfectants constitute the cell lines transfected with vector alone.

Duplex RT-PCR. Total RNA from fresh tissues and cell lines was isolated with TRIzol reagent according to the manufacturer's protocol (Invitrogen). Following DNase I treatment in order to eliminate genomic DNA contamination, $3 \mu \mathrm{g}$ of total RNA per sample were used to generate cDNA using a superscript enzyme (Superscript II RT enzyme, Invitrogen). The resulting cDNAs were subsequently amplified, analyzed, and quantified as previously described (23). Primer sequences, PCR conditions and the amplified lengths have been described (24). Glyceraldehyde-3-phosphate dehydrogenase (GAPDH) was used as a reference gene. Linear amplification range for each gene was determined by preparing 6 similar reactions but amplified by different number of cycles $(26,29,32,35$, 38 , and 41 cycles). For all genes, the chosen cycle was in the exponential phase of the PCR amplification.

Immunohistochemistry. HOXB7 and Ki67 immunostaining was performed using the streptavidin-biotin peroxidase complex method. Briefly, after dewaxing and hydration in graded alcohol solutions, the sections were treated with $3 \%$ $\mathrm{H}_{2} \mathrm{O}_{2}$, followed by antigen retrieval with $10 \mathrm{mM}$ citric acid pH 6.0 in a pressure cooker. After washing with phosphatebuffered saline (PBS), the sections were treated with $1 \%$ bovine serum albumin (BSA) in PBS for $1 \mathrm{~h}$, and then incubated with polyclonal rabbit anti-HOXB7 (Zymed Lab., San Francisco, CA, USA) diluted 1:200 or monoclonal mouse anti-Ki67 (Dako Corp., Carpenteria, CA, USA) diluted 1:100, followed by the LSAB method (LSAB+ System-HRP kit, Dako). Reactions were developed by incubating the sections with $0.6 \mathrm{mg} / \mathrm{ml} \mathrm{3,3'-diaminobenzidine} \mathrm{tetrahydrochloride}$ (Sigma-Aldrich) containing $0.01 \% \mathrm{H}_{2} \mathrm{O}_{2}$. The control reactions were performed by the omission of the primary antibodies. The percentage of nuclear-positive cells was calculated with the aid of an image computer analyzer (Kontron 400, Carl Zeiss, Germany).

Western blot analysis. Cells were washed with cold PBS and lysed in RIPA buffer (50 mM Tris- $\mathrm{HCl}$ pH 7.4, $150 \mathrm{mM}$ $\mathrm{NaCl}, 1 \mathrm{mM}$ EDTA, $1 \%$ NP-40, $1 \%$ deoxycholic acid, $0.5 \%$ sodium dodecyl sulfate, $1 \mathrm{mM}$ phenymethylsulfony fluoride, $1 \mathrm{mM}$ N-ethylmaleimide, $1 \mathrm{mM}$ dithiothreitol, $10 \mu \mathrm{g} / \mathrm{ml}$ soybean trypsin inhibitor, $1 \mu \mathrm{g} / \mathrm{ml}$ leupeptin and $1 \mu \mathrm{g} / \mathrm{ml}$ aprotinin). After centrifugation, protein concentrations were measured using a protein assay according to the manufacturer's instructions (Bio-Rad Protein Assay, Bio-Rad, Hercules, CA). Eighty $\mu \mathrm{g}$ of total protein per sample were resolved in a $10 \%$ sodium dodecyl sulphate polyacrylamide gel electrophoresis (SDS-PAGE) under reducing conditions, and transferred to nitrocellulose membranes. The membranes were blocked for $2 \mathrm{~h}$ with $10 \%$ non-fat dry milk in PBS containing $0.1 \%$ Tween-20, rinsed in the same buffer, and incubated for $1 \mathrm{~h}$ with anti-HOXB7 antibodies diluted 1:200 or with anti- $\beta$-actin antibodies (Sigma-Aldrich) diluted 1:70,000 in 5\% milk in PBS. After washing, the membranes were developed using an Enhanced Chemiluminescent Western blot kit (GE Healthcare, Vienna, Austria).

Bromodeoxyuridine-labeling ( $\mathrm{BrdU})$ index. Cells were plated in 8-well chamber slides at a density of 30,000 cells per well in $500 \mu \mathrm{l}$ of medium containing $10 \%$ FBS. After $16 \mathrm{~h}$, the cells were washed with PBS and cultured in serum-free medium for an additional $24 \mathrm{~h}$. Following serum starvation, the medium was replaced by $10 \%$ FBS medium. Proliferation rates were determined $24 \mathrm{~h}$ after incubation by measuring BrdU incorporation into DNA. Briefly, BrdU antigen was added to the cultures and kept for $1 \mathrm{~h}$ at $37^{\circ} \mathrm{C}$ in $5 \% \mathrm{CO}_{2}$. After incubation, cells were washed in PBS and fixed in $70 \%$ ethanol for $1 \mathrm{~h}$. BrdU incorporation in proliferating cells was estimated using an immunohistochemical analysis kit (GE Healthcare). The BrdU-labeling index, expressed as the percentage of cells labeled with BrdU, was determined by 


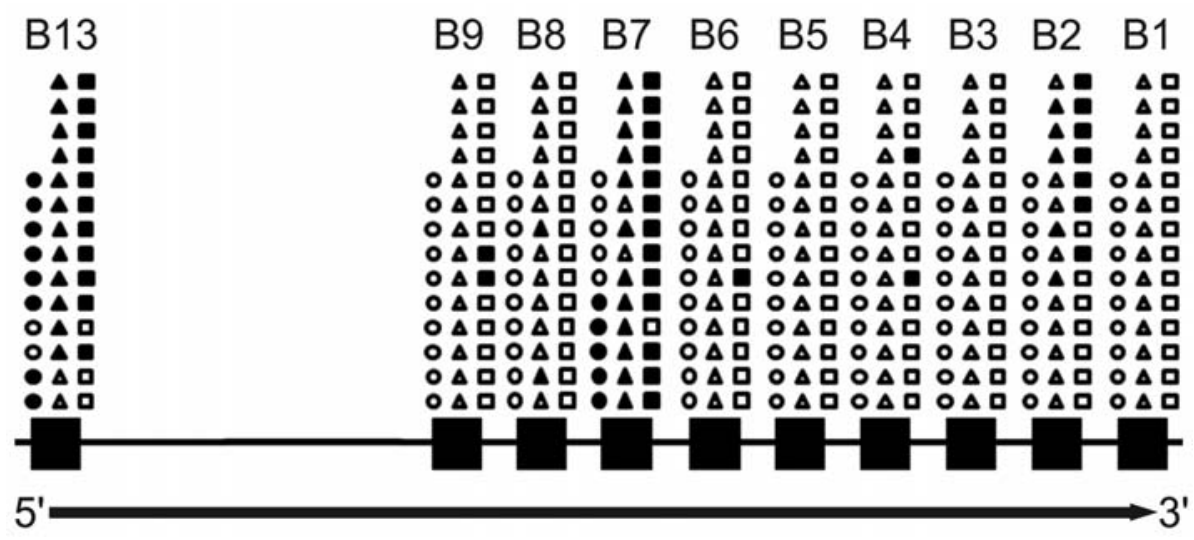

Figure 1. Diagram of HOXB gene expression in normal oral mucosas and OSCCs. Each symbol represents one specific sample, and their positions are preserved throughout the image. Circles represent normal oral mucosa derived of patients without contact with main oral cancer risk factors, and triangles and squares represent respectively normal oral mucosa and OSCC from the same patient. Open symbols indicate silent (inactive) HOXB genes, whereas closed symbols indicate active genes. Most of the HOX genes belonging to cluster B are silenced in the oral cavity, with exception of $\mathrm{HOXB7}$ and $\mathrm{HOXB13.}$

counting 1,500 cells in 3 independent reactions using the Kontron 400 image analysis system (Zeiss).

Ki67 index. Cells were cultured in culture chamber slides at $37^{\circ} \mathrm{C}$ in humidified air containing $5 \% \mathrm{CO}_{2}$ for $24 \mathrm{~h}$. After cellular synchronism and cell cycle induction as described above, the cells were fixed in $70 \%$ ethanol for $1 \mathrm{~h}$ and washed with PBS. Cells were then treated with $1 \%$ BSA diluted in PBS for $1 \mathrm{~h}$, incubated with monoclonal antibodies against Ki67, and followed by the ABC method (StrepABC Complex/HRP, Dako). Reactions were developed with $0.6 \mathrm{mg} / \mathrm{ml} \mathrm{DAB}$ containing $0.01 \% \mathrm{H}_{2} \mathrm{O}_{2}$. The $\mathrm{Ki} 67$ index was calculated using an image analysis system by counting labeled nuclei of 1,500 cells in 3 independent reactions, and expressed as percentage of Ki67-positive cells.

Apoptosis analysis. Apoptosis index was determined by annexin V-FITC labeling (25). Briefly, cells were harvested, washed with PBS and resuspended in the binding buffer (10 mM HEPES pH 7.4, $150 \mathrm{mM} \mathrm{NaCl}, 5 \mathrm{mM} \mathrm{KCl}, 1 \mathrm{mM}$ $\mathrm{MgCl}_{2}$, and $1.8 \mathrm{mM} \mathrm{CaCl}$ ) containing annexin V-FITC at 1:500. After 20 min of incubation in the dark at room temperature, cells were also stained with propidium iodide (PI, Sigma-Aldrich). Apoptosis was analyzed on a FACScalibur flow cytometer equipped with an argon laser (BectonDickinson, San Jose, CA, USA) and quantified as the number of annexin V-FITC positive and PI negative cells divided by the total number of cells. A minimum of 10,000 events was analyzed in each sample.

Small interference RNA (siRNA) mediating HOXB7 silence. To determine the role of endogenous HOXB7, we examined the effect of HOXB7 siRNA transfection on SCC9 cell line proliferation and survival. The 25-mer RNA molecules were chemically synthesized, annealed and purified by the manufacturer (Invitrogen). Three sequenced targeting HOXB7 (NC_000017) were used, corresponding to nucleotides 106-130 (5' GAG TTC ATT GTA TTA TGC GAA TGC T'), 342-366 (5' TCG AGC CGA GTT CCT TCA ACA TGC A 3'), and 3047-3071 (5' TCT GCC TCA CGG AAA GAC AGA
TCA A $3^{\prime}$ ). In essence, cells grown to $50 \%$ confluence were transfected with $100 \mathrm{nM}$ of a mixture containing equal parts of the 3 HOXB7 siRNAs using a liposome method according to the manufacturer's instructions (Lipofectamine 2000, Invitrogen). In parallel, to act as negative controls, cell were transfected with a non-specific siRNA or with the transfectant reagent (mock transfection). Forty-eight hours after transfection, the efficacy of the HOXB7 knock down was determined by duplex-RT-PCR and Western blot analysis. Proliferation and survival assays were performed as described above.

Statistical analysis. To analyze HOXB7 mRNA and protein levels between groups, Kruskal-Wallis multiple-comparison test was used. Student's t-test (bi-caudal) was used to compare the proliferation of transfectant cell lines and of SCC9 under siRNA treatment. Spearman rank correlation test (rs) was assessed to verify the association between immunohistochemical expression of HOXB7 and Ki67. Correlations between immunohistochemical expression of HOXB7 and clinicopathological parameters of the tumors for frequency data in contingency tables were performed by Fisher's exact test, and for continuous variables by non-parametric MannWhitney U test.

\section{Results}

Expression of $\mathrm{HOXB}$ genes in normal oral mucosa and OSCC. Expression of the HOXB cluster genes was initially detected using total RNA extracted from normal and neoplastic tissues through duplex RT-PCR with specific oligonucleotides designed against each of the 10 genes of the HOXB locus and GAPDH. General analysis indicated that HOXB network is inactive in the oral cavity (Fig. 1). Four out of 10 genes (HOXB1, HOXB3, HOXB5 and HOXB8) were silenced in all samples from both normal oral mucosa and OSCC. In addition, normal oral mucosa samples from patients without recognized OSCC-risk factors showed no mRNA transcripts for HOXB2, HOXB4, HOXB6, and HOXB9. HOXB7 and HOXB 13 were expressed by 50 and $80 \%$, respectively, of the 


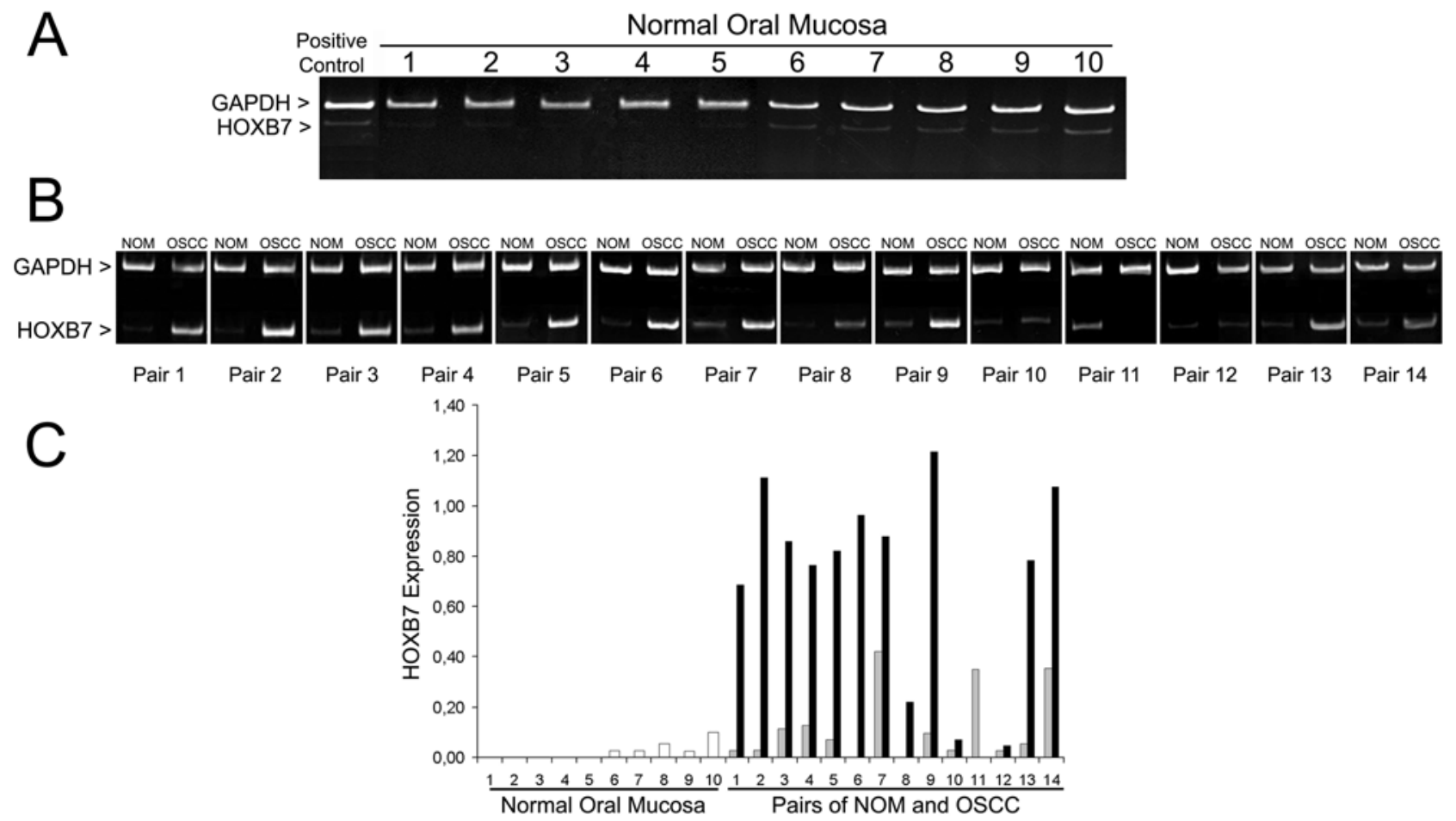

Figure 2. HOXB7 is overexpressed in OSCCs. Total RNA from fresh samples was isolated, and cDNA synthesized by RT. (A) Duplex RT-PCR analysis of HOXB7 on the normal oral mucosa from patients without contact with the main risk factors to oral cancer; and (B) HOXB7 analysis on the pairs of OSCC and adjacent normal oral mucosa from the same patient. (C) Densitometric analysis of the HOXB7 bands demonstrated a significant higher expression in OSCCs compared to normal oral mucosas, independent of the source $(\mathrm{p}<0.001$ when compared to normal oral mucosa from patients without recognized OSCC-risk factors, and $\mathrm{p}<0.05$ when compared to normal oral mucosa from patients with OSCC).

samples from patients without recognized OSCC-risk factors, and both were expressed by $85.7 \%$ of the normal oral mucosa samples from patients with OSCC. In OSCCs, with exception of the members HOXB2, HOXB7 and HOXB13, the expression levels of $\mathrm{HOXB}$ genes were very low and detected in few samples.

Considering the intensity levels of gene expression, as represented by the densitometric ratio of the optical density of target transcript/GAPDH bands, we identified that the gene HOXB7 is significantly more expressed by OSCC samples compared to normal oral mucosa samples from patients without recognized OSCC-risk factors $(\mathrm{p}<0.001)$ and from patients with OSCC $(\mathrm{p}<0.05)$ (Fig. 2). The levels of HOXB2 were also significantly higher in OSCC samples than in normal oral mucosa samples from patients without recognized OSCC-risk factors $(\mathrm{p}<0.05)$, but similar to the matched normal oral mucosa samples (data not shown). No differences on HOXB13 expression were found.

To confirm HOXB7 higher expression on OSCC samples compared to normal oral mucosa, we performed immunohistochemical analysis. Immunoreactivity for HOXB7 was observed as a nuclear stain restricted to the basal layer in the normal oral mucosas, whereas a broad positivity with variable distribution and intensity was found in the OSCCs (Fig. 3A and B). As expected, OSCCs showed HOXB7labeling index significantly higher than the mean of normal oral mucosa group ( $<<0.001$; Fig. $3 \mathrm{C})$. The percentage of HOXB7positive cells in normal oral mucosa group ranged between
0 and $18 \%$, with a median of $5.7 \%$ (mean $8.01 \pm 6.94 \%$ ), whereas for OSCC group ranged between 10 and $63 \%$, with a median of $29.1 \%$ (mean $32.37 \pm 15.66 \%$ ).

HOXB7 overexpression promotes a proliferative phenotype in OSCC cells. To determine whether HOXB7 overexpression contributes to the tumorigenic phenotype and, more specifically, to proliferation and apoptosis, we next overexpressed HOXB7 in the HaCAT epithelial cell line, which does not show detectable endogenous levels of this protein. Stable HOXB7 and control transfectants were generated and examined for HOXB7 protein levels. Three stable HOXB7-overexpressing clones (HaCAT-HOXB7) and 3 control clones (HaCAT-control) were chosen for further analyses (Fig. 4A).

HOXB7-overexpressing cells showed a statistically significant increase in proliferation, as assessed by measuring BrdU incorporation into DNA and Ki67 expression (Fig. 4B and C). Consistently, the analysis of OSCC samples depicted in Fig. 1 for Ki67 immunohistochemical expression demonstrated a statistically significant correlation between percentage of HOXB7-positive cells and Ki67-positive cells ( $r s=0.79, p<0.006)$. In order to verify whether the endogenous function of $\mathrm{HOXB7}$ is to promote proliferation, it was next knocked down in OSCC cells expressing high levels of HOXB7 (SCC9 cells). Specific-stranded RNA oligonucleotides against $\mathrm{HOXB} 7$ or negative RNA control were transfected into SCC9 cells. When HOXB7-specific 

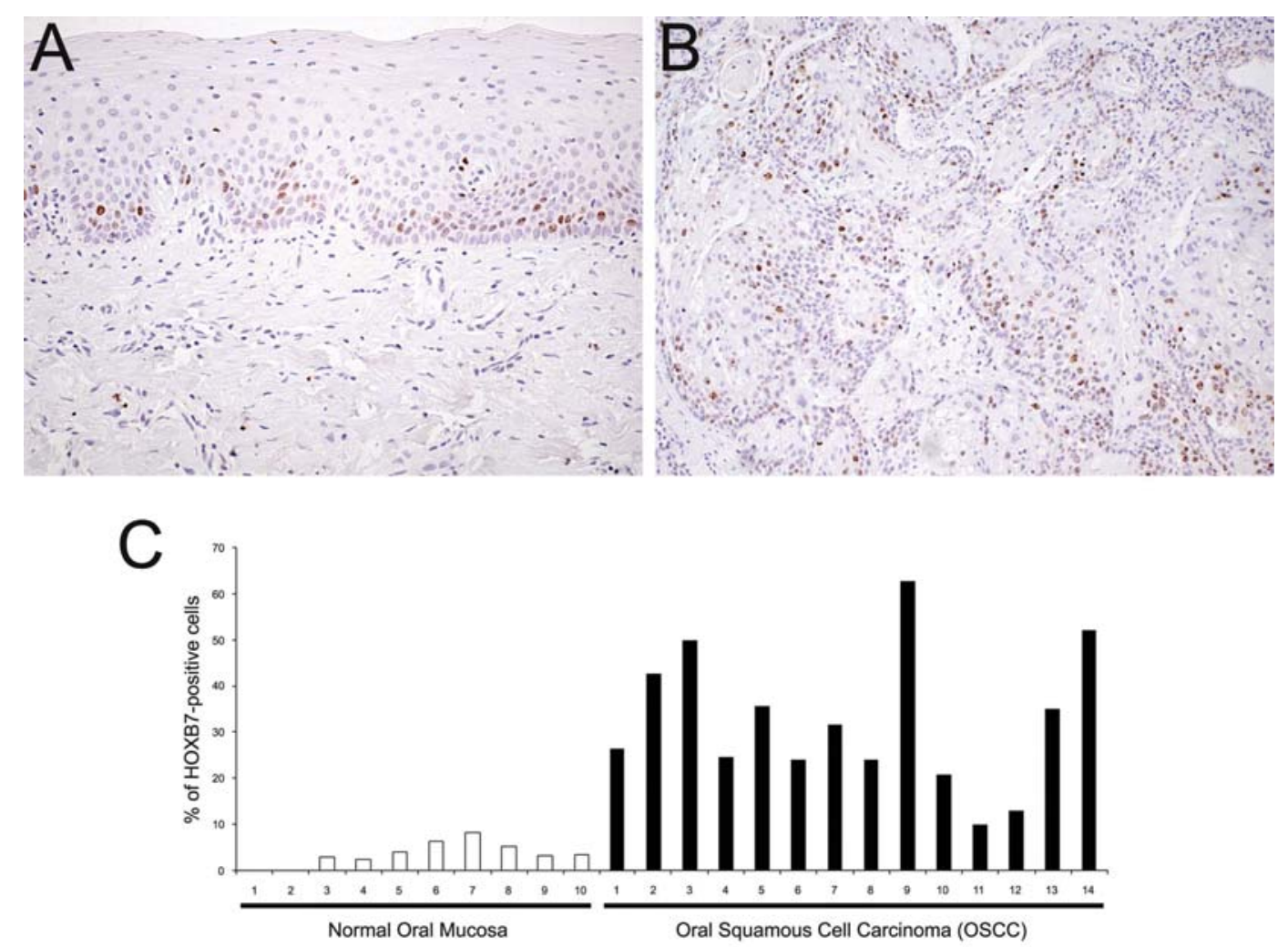

Figure 3. Immunohistochemical detection of HOXB7 in normal oral mucosa and OSCC. Representative samples of normal oral mucosa (A) and OSCC (B) of this study. In the normal mucosa, HOXB7 expression was clearly limited to the nucleus of the epithelial cells located in the basal and suprabasal layers, whereas in the OSCCs HOXB7-positive cells were broadly observed in the tumor (original magnification x200). (C) Percentage of HOXB7-positive cells was significantly higher in OSCCs than in normal oral mucosas $(\mathrm{p}<0.001)$.
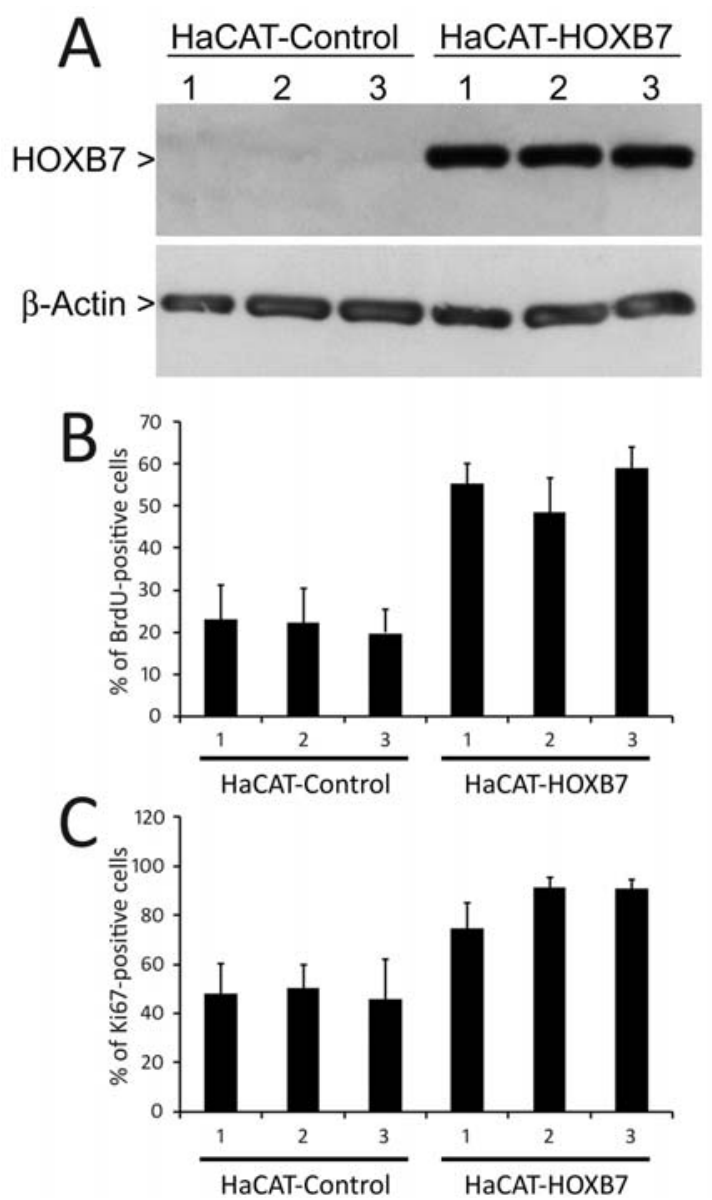

oligonucleotides were used, a rapid downregulation of HOXB7 mRNA and protein was observed (Fig. 5A and B). The decrease in HOXB7 levels resulted in a concomitant decrease in proliferation. Cell proliferation assays (BrdU incorporation and Ki67 expression) showed a statistically significant decrease in proliferation when HOXB7 was downregulated with siRNA (Fig. 5C and D). The number of annexin V-FITC-positive cells was very low in both HOXB7overexpressing cells and SCC9 cells with HOXB7 downregulated by siRNAs, and no significant differences from controls were observed (data not shown).

HOXB7 expression correlates with clinicopathological features of OSCCs. To investigate whether HOXB7 expression is associated with clinicopathological features of oral cancer and with the proliferative potential of the tumor cells, we performed immunohistochemistry on 35 human OSCCs to examine HOXB7 expression concurrent with Ki67 proliferative

Figure 4. Overexpression of HOXB7 induces cellular proliferation. (A) Representative Western blot analysis of HOXB7 in HaCAT-control and HaCAT-HOXB7 transfectants, revealing an increase in HOXB7 levels in HOXB7 overexpressing HaCAT cells. Assays measuring BrdU incorporation (B) and Ki67 expression (C) demonstrate that HOXB7 overexpressing cell lines have a statistically significant increase in proliferation as compared to control cells (for BrdU index $\mathrm{p}<0.0001$ between groups, and for Ki67 index $\mathrm{p}<0.001$ between groups). The labeling index of BrdU and Ki67 correspond to the mean percentage of positive cells of 3 experiments for each cell line. 


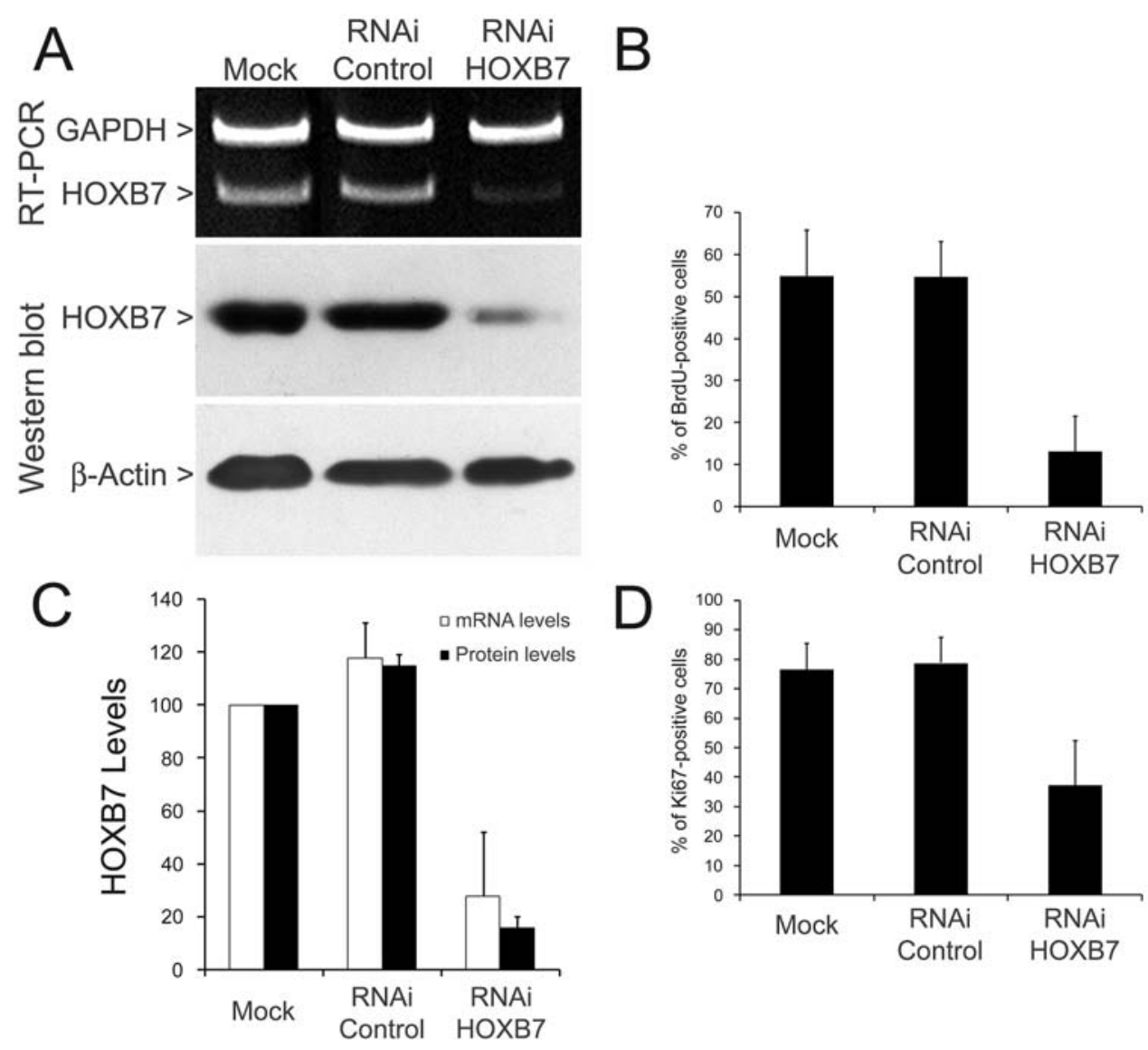

Figure 5. Inhibition of HOXB7 by siRNA in SCC9 cells decreases cellular proliferation. (A) siRNA against HOXB7 decreases HOXB7 levels as revealed by representative duplex RT-PCR and Western blot analysis (HOXB7 and B-actin). (B) Densitometric analysis of 3 experiments of siRNA demonstrating a significant inhibition on both HOXB7 mRNA and protein levels. BrdU incorporation (C) and Ki67 immunocytochemical expression (D) assays showed a statistically significant decrease in proliferation when HOXB7 was downregulated via siRNA (for BrdU index p<0.001 and for Ki67 index p<0.005). Data correspond to the mean percentage of positive cells of 3 experiments.
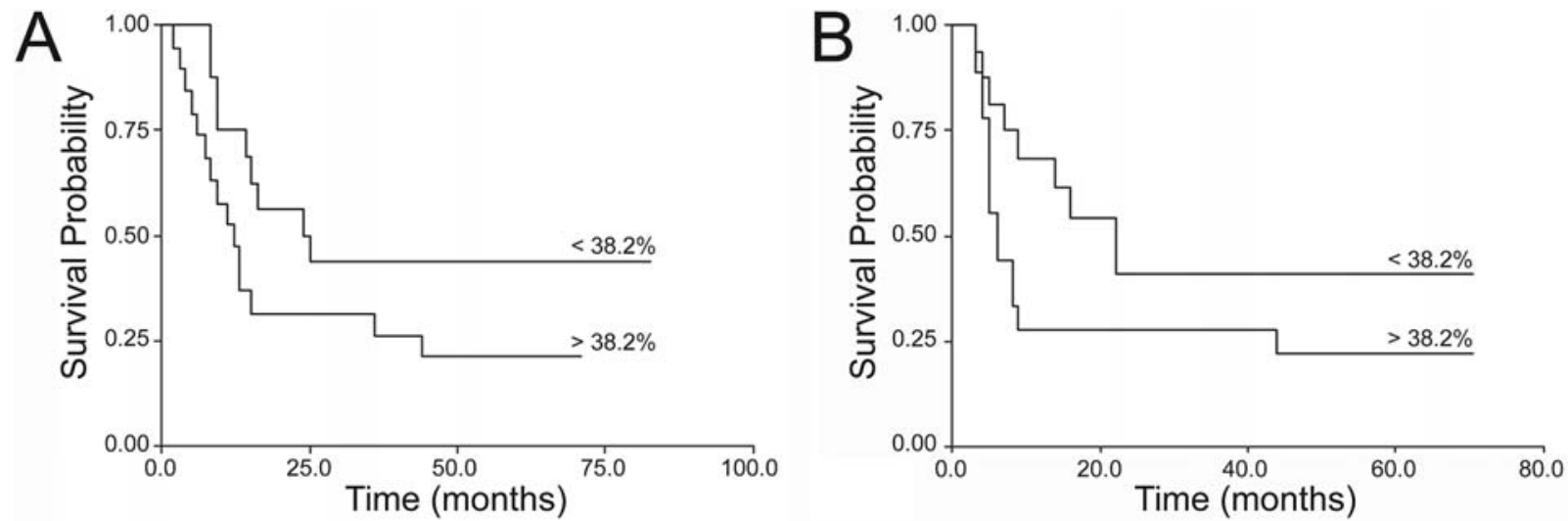

Figure 6. HOXB7 expression is associated with poor prognosis. (A) Curves of overall survival and (B) disease-free survival for the patients of this study. The median value of HOXB7-positive cells was employed to divide tumors into 2 groups, below and above the respective medians, exhibiting low $(<38.2 \%$ of positive cells) and high ( $>38.2 \%$ of positive cells) HOXB7 levels. Patients with high number of HOXB7-positive tumor cells had shorter global survival period $(\mathrm{p}=0.08)$ and disease-free survival after treatment $(\mathrm{p}=0.10)$ than patients with low number of HOXB7-positive tumor cells.

marker. Parallel slides were analyzed for HOXB7 and Ki67 expression. The clinicopathological correlations with the expression of HOXB7 are listed in Table I. Elevated amount of HOXB7-positive cells in the tumor was correlated with T stage $(\mathrm{p}=0.06), \mathrm{N}$ stage $(\mathrm{p}=0.07)$, and disease stage $(\mathrm{p}=0.09)$. Regional (cervical) recurrence was observed in only 3 patients of our group, but all demonstrated tumors classified as having high expression of HOXB7. Expression of HOXB7 was not associated with age, gender, smoking habit, alcohol consumption, tumor cellular differentiation, and local or distance tumor recurrence. We found that tumors that overexpressed HOXB7 showed statistically significant increase 
Table I. Relationship between HOXB7 immunohistochemical expression and clinicopathological variables of the OSCCs.

\begin{tabular}{|c|c|c|c|}
\hline \multirow[b]{2}{*}{ Parameter } & \multicolumn{2}{|c|}{$\%$ of $\mathrm{HOXB} 7$ positive cells } & \multirow[b]{2}{*}{ P-value } \\
\hline & $\begin{array}{l}<38.2 \\
\mathrm{n}(\%)\end{array}$ & $\begin{array}{l}>38.2 \\
\mathrm{n}(\%)\end{array}$ & \\
\hline
\end{tabular}

Age

$\leq 60$ years

$>60$ years

$10 \quad(62.5) \quad 9(47.36)$

0.37

Gender

Male

Female

$6 \quad(37.5) \quad 10(52.64)$

0.90

Smoking habit

$\begin{array}{lrrr}\text { No } & 4 & (25) & 2(10.52) \\ \text { Yes } & 12 & (75) & 17(89.48)\end{array}$

Alcohol consumption

No

Yes

$7(43.75) \quad 8 \quad(42.1)$

$9(56.25) \quad 11 \quad$ (57.9)

T Stage

$\mathrm{T} 1+\mathrm{T} 2$

$\mathrm{T} 3+\mathrm{T} 4$

$10 \quad(62.5) \quad 6(31.57)$

$6 \quad(37.5) \quad 13(68.43)$

N Stage

No

$\mathrm{N}+$

$9(56.25) \quad 5(26.31)$

7 (43.75) $14(73.69)$

Disease stage

Stage I + II

Stage III + IV

$9(56.25) \quad 6(31.57)$

7 (43.75) 13 (68.43)

Differentiation $^{\mathrm{a}}$

\begin{tabular}{|c|c|c|}
\hline WD & $7(43.75)$ & $5(26.31)$ \\
\hline MD & $7(43.75)$ & $10(52.63)$ \\
\hline $\mathrm{PD}+\mathrm{U}$ & $2(12.5)$ & $4(21.06)$ \\
\hline
\end{tabular}

Local recurrence

No

Yes

$9(56.25) \quad 15(78.94)$

$7(43.75) \quad 4(21.06)$

Regional recurrence

No

Yes

$16 \quad(100) \quad 16(84.21)$

$0 \quad 3(15.79)$

0.09

Distance recurrence

$\begin{array}{lrrr}\text { No } & 14 & (87.5) & 15(78.94) \\ \text { Yes } & 2 & (12.5) & 4(21.06)\end{array}$

Ki67-positive cells

$\begin{array}{rrrr}\leq 33.6 \% & 12 & (75) & 6(31.57) \\ >33.6 \% & 4 & (25) & 13(68.43)\end{array}$

aDifferentiation: WD, well differentiated; MD, moderately differentiated; PD, poorly differentiated; and U, undifferentiated. in the percentage of Ki67-positive cells compared with low expressing tumors (Table I). Furthermore, there was a significant correlation between percentage of HOXB7- and Ki67-positive cells in the studied tumors ( $\mathrm{rs}=0.48$, $\mathrm{p}=0.003$ ). Patients whose tumors contained high number of HOXB7positive cells had shorter overall survival than patients with low number of this protein ( $\mathrm{p}=0.08$; Fig. 6A). Similarly, patients with tumors showing high HOXB7 expression had shorter disease-free survival after treatment $(\mathrm{p}=0.10$, Fig. $6 \mathrm{~B})$. Taken together, these data demonstrate that overexpression of HOXB7 in oral cancer is associated with several clinicopathological features of the tumors, including elevated proliferative potential and worsened survival.

\section{Discussion}

During development, tissue-specific mechanisms exist for the expansion of precursor populations prior to differentiation, after which proliferation is largely halted. Members of the HOX family of homeobox genes play specific roles in both processes during development, first by inducing cell proliferation and later by conferring cell fate, consequently defining regional identity of the tissues (26). In adults, most of the HOX genes are silent, with exception of few members that are constitutively expressed in limited tissues and organs, such as hematopoietic tissue (27), pregnant mammary glands (28), and uterus during the menstrual cycle (29). In all those tissues, HOX gene expression is identified in proliferating and/or differentiating cells. Since the first description of altered expression in leukemias (30), dysregulation of HOX gene expression is commonly observed in human cancers. Typically, those genes that are overexpressed in cancers show normal expression patterns that are restricted to the undifferentiated or proliferative cells, whereas those that are lost in cancer are expressed in fully differentiated cells (31). Moreover, the classical mechanism of sequential activation of HOX genes is lost in cancers, and specific functions are played by single genes (6). Although these powerful developmental regulators are known to affect numerous important processes in embryogenesis and tumorigenesis $(3,32)$, a causal role for HOX genes in tumor initiation and/or progression remains controversial.

To identify differentially expressed genes, this study analyzed HOX genes belonging to cluster B in pairs of histologically normal oral mucosa and tumor, and in samples of normal oral mucosa obtained of patients not exposed to risk factors related to OSCC. In normal oral mucosa derived from patients without risk factors to oral cancer, only HOXB7 and HOXB13 were expressed, whereas 5 out of 14 samples of the normal oral mucosa from OSCC patient group expressed HOXB2, suggesting that alterations on HOXB2 expression can precede any histological alteration suggestive of malignancy. Further investigation to address the role of HOXB2 on oral carcinogenesis is needed. Besides those genes, few OSCC samples also expressed HOXB4, HOXB6 and HOXB9. However, among the genes detected in this study, only the expression of HOXB7 was significantly higher in OSCC compared with normal oral mucosa. In addition to the mRNA quantification, we showed that HOXB7 protein expression is limited to the basal cells of the normal epithelium, while that oral cancers show strong and broad immunoreactivity in 
the neoplastic cells. As expected, the number of HOXB7positive cells was significantly higher in OSCCs than that of controls.

HOXB7 plays a critical role in expanding progenitor cells during normal development via its stimulation of proliferation (18) and upregulation of HOXB7 activity is observed in several tumors, suggesting that when expressed out of context, it may contribute to tumorigenesis by reinstating properties normally conveyed on developing cells $(33,34)$. Since HOXB7 is important for the expansion of progenitor cell populations during embryonic development, an increase in proliferation coupled with a decrease in apoptosis would have dire consequences in an adult epithelium. Indeed, HOXB7 contributes to tumor cell proliferation both in breast cancer and in melanomas, in which it is also implicated in angiogenesis $(16,17,33,34)$. Moreover, overexpression of HOXB7 induced anchorage-independent growth of mammary epithelial cells $(17,34)$ and NIH3T3 fibroblasts (35). Recently, Chen and collaborators (18) demonstrated that overexpression of HOXB7 is insufficient to cause tumor formation in the mammary gland of transgenic mice, but revealed a dual effect of HOXB7 during tumor formation and progression. Under HER-2/neu background, overexpression of HOXB7 inhibited tumor onset while promoted tumor growth and metastasis. Taken together, these data strongly support an oncogenic role for HOXB7.

Overexpression of HOXB7 was observed in esophageal and oral cancers $(8,15)$. HOXB7 was expressed in $\sim 58 \%$ of the tumors arising in the esophagus but not in the normal esophageal mucosa (8). In the oral cavity, HOXB7 expression was detected in normal, dysplastic and neoplastic samples, with levels significantly higher in OSCCs than in normal mucosas and dysplasias (15). The results presented here clearly demonstrate that HOXB7 overexpression accelerated cell cycle progression, and a decrease in HOXB7 levels slowed the progression of cells through the cell cycle. In addition, HOXB7 levels correlated with the proliferative potential of the oral tumor cells, as revealed in vivo by significant correlation between HOXB7 and Ki67 levels. As a transcriptional factor with upstream activators, downstream unknown targets and functional partners, various functions of $\mathrm{HOXB} 7$ can impinge on normal cell proliferation. It is known that HOXB7 induces the expression of basic fibroblast growth factor (bFGF) in vitro in breast cancer and melanoma cell lines $(16,17,33)$ and in vivo in transgenic mice (18). Our study also revealed that modulation of HOXB7 levels had no dramatic effect on cell death. Similarly, Chen et al (18) demonstrated no effect of HOXB7 overexpression on cell death in breast cancers of HOXB7-HER2/neu dual transgenic mice. Furthermore, an effect on survival of HOXB7-overexpressing breast cells was only observed after ionizant irradiation, due to an increase in the DNA repair rate (19).

Most importantly, we have further demonstrated that abundant presence of HOXB7-positive cells leads to a more aggressive behavior of the OSCCs. Tumors with high percent of HOXB7-positive cells tended to be in advanced clinical stage of the disease (higher $\mathrm{T}$ stage, positive lymph node metastasis and late disease stage), and survival analysis showed that patients whose tumors had high number of HOXB7-positive cells had a shortened overall survival and disease-free survival. Poor prognosis associated with high levels of HOXB7 was independent of other factors, suggesting that HOXB7 expression may be an important independent prognostic indicator of poor survival for oral cancer patients. In support of this concept, a recent study showed that overexpression of HOXB7 induces in vitro epithelial-mesenchymal transition, which is accompanied by acquisition of aggressive properties of tumorigenicity such as migration and invasion, and forms in vivo aggressive invasive tumors that are well vascularized (34). Moreover, it was described that HOXB7 overexpression in the mammary glands induces poorly differentiated tumors with high mitotic index and elevate potential to induce micrometastasis in the lungs (18). Together these results reinforce ours and other clinical data evidencing the importance of HOXB7 for the tumor behavior.

In conclusion, the evidence presented in this report that HOXB7 is differentially expressed between normal oral tissues and OSCCs, induces proliferative activity, and its expression correlates with features related to poor prognosis of OSCC patients, strongly support a role for this gene in oral carcinogenesis.

\section{Acknowledgments}

This work was supported by grants from Fundação de Amparo a Pesquisa do Estado de São Paulo-FAPESP, São Paulo, Brazil; and Conselho Nacional de Desenvolvimento Científico e Tecnológico-CNPq, Brasília, Brazil. M.F.S. Destro was supported by Fundação de Amparo a Pesquisa do Estado de São Paulo-FAPESP, São Paulo, Brazil, and C.C. Bitu is supported by Conselho Nacional de Desenvolvimento Científico e Tecnológico-CNPq, Brasília.

\section{References}

1. Cantile M, Schiavo G, Terracciano L and Cillo C: Homeobox genes in normal and abnormal vasculogenesis. Nutr Metab Cardiovasc Dis 18: 651-658, 2008.

2. Abate-Shen C: Deregulated homeobox gene expression in cancer: cause or consequence? Nat Rev Cancer 2: 777-785, 2002.

3. Chen H and Sukumar S: Role of homeobox genes in normal mammary gland development and breast tumorigenesis. J Mammary Gland Biol Neoplasia 8: 159-175, 2003.

4. Chen $\mathrm{H}$ and Sukumar S: HOX genes: emerging stars in cancer. Cancer Biol Ther 2: 524-525, 2003.

5. Del Bene F and Wittbrodt J: Cell cycle control by homeobox genes in development and disease. Semin Cell Dev Biol 16: 449-460, 2005.

6. Samuel $\mathrm{S}$ and Naora $\mathrm{H}$ : Homeobox gene expression in cancer: insights from developmental regulation and deregulation. Eur $\mathrm{J}$ Cancer 41: 2428-2437, 2005.

7. Cillo C, Faiella A, Cantile M and Boncinelli E: Homeobox genes and cancer. Exp Cell Res 10: 1-9, 1999.

8. Chen KN, Gu ZD, Ke Y, Li JY, Shi XT and Xu GW: Expression of $11 \mathrm{HOX}$ genes is deregulated in esophageal squamous cell carcinoma. Clin Cancer Res 11: 1044-1049, 2005.

9. Coletta RD, Jedlicka P, Gutierrez-Hartmann A and Ford HL: Transcriptional control of the cell cycle in mammary gland development and tumorigenesis. J Mammary Gland Biol Neoplasia 9: 39-53, 2004.

10. Lopez R, Garrido E, Vazquez G, Pina P, Perez C, Alvarado I and Salcedo M: A subgroup of HOX Abd-B gene is differentially expressed in cervical cancer. Int J Gynecol Cancer 16: 1289-1296, 2006.

11. Maeda K, Hamada J, Takahashi Y, Tada M, Yamamoto Y, Sugihara T and Moriuchi T: Altered expressions of HOX genes in human cutaneous malignant melanoma. Int J Cancer 114: 436-441, 2005. 
12. Maroulakou IG and Spyropoulos DD: The study of HOX gene function in hematopoietic, breast and lung carcinogenesis. Anticancer Res 23: 2101-2110, 2003.

13. McGonigle GJ, Lappin TR and Thompson A: Grappling with the HOX network in hematopoiesis and leukemia. Front Biosci 13: 4297-4308, 2008.

14. Waltregny D, Alami Y, Clausse N, De Leval J and Castronovo V: Overexpression of the homeobox gene HOXC8 in human prostate cancer correlates with loss of tumor differentiation. Prostate 50: $162-169,2002$

15. Hassan NM, Hamada J, Murai T, Seino A, Takahashi Y, Tada M, Zhang X, Kashiwazaki H, Yamazaki Y, Inoue N and Moriuchi T: Aberrant expression of HOX genes in oral dysplasia and squamous cell carcinoma tissues. Oncol Res 16: 217-224, 2006.

16. Care A, Silvani A, Meccia E, Mattia G, Peschle C and Colombo MP: Transduction of the $\mathrm{SkBr} 3$ breast carcinoma cell line with the HOXB7 gene induces bFGF expression, increases cell proliferation and reduces growth factor dependence. Oncogene 16: 3285-3289, 1998.

17. Care A, Silvani A, Meccia E, Mattia G, Stoppacciaro A, Parmiani G, Peschle C and Colombo MP: HOXB7 constitutively activates basic fibroblast growth factor in melanomas. Mol Cell Biol 16: 4842-4851, 1996.

18. Chen H, Lee JS, Liang X, Zhang H, Zhu T, Zhang Z, et al: Hoxb7 inhibits transgenic HER-2/neu-induced mouse mammary tumor onset but promotes progression and lung metastasis. Cancer Res 68: 3637-3644, 2008.

19. Rubin E, Wu X, Zhu T, Cheung JC, Chen H, Lorincz A, Pandita RK, Sharma GG, Ha HC, Gasson J, Hanakahi LA Pandita TK and Sukumar S: A role for the HOXB7 homeodomain protein in DNA repair. Cancer Res 67: 1527-1535, 2007.

20. Anneroth G, Batsakis J and Luna M: Review of the literature and a recommended system of malignancy grading in oral squamous cell carcinomas. Scand J Dent Res 95: 229-249, 1987.

21. Coletta RD, Christensen K, Reichenberger KJ, Lamb J, Micomonaco D, Huang L, Wolf DM, Müller-Tidow C, Golub TR, Kawakami K and Ford HL: The Six1 homeoprotein stimulates tumorigenesis by reactivation of cyclin A1. Proc Natl Acad Sci USA 101: 6478-6483, 2004.

22. Yaron Y, McAdara JK, Lynch M, Hughes E and Gasson JC: Identification of novel functional regions important for the activity of HOXB7 in mammalian cells. J Immunol 166: 5058-5067, 2001.

23. Martelli-Junior H, Cotrim P, Graner E, Sauk JJ and Coletta RD Effect of transforming growth factor-beta1, interleukin-6, and interferon-gamma on the expression of type I collagen, heat shock protein 47, matrix metalloproteinase (MMP)-1 and MMP-2 by fibroblasts from normal gingiva and hereditary gingival fibromatosis. J Periodontol 74: 296-306, 2003.
24. Yamamoto M, Takai D and Yamamoto F: Comprehensive expression profiling of highly homologous 39 hox genes in 26 different human adult tissues by the modified systematic multiplex RT-pCR method reveals tissue-specific expression pattern that suggests an important role of chromosomal structure in the regulation of hox gene expression in adult tissues. Gene Expr 11: 199-210, 2003.

25. Zecchin KG, Seidinger AL, Chiaratti MR, Degasperi GR, Meirelles FV, Castilho RF and Vercesi AE: High Bcl-2/Bax ratio in Walker tumor cells protects mitochondria but does not prevent $\mathrm{H}_{2} \mathrm{O}_{2}$-induced apoptosis via calcineurin pathways. J Bioenerg Biomembr 39: 186-194, 2007.

26. Bondos SE, Tan XX and Matthews KS: Physical and genetic interactions link hox function with diverse transcription factors and cell signaling proteins. Mol Cell Proteomics 5: 824-834, 2006.

27. Taghon T, Thys K, De Smedt M, Weerkamp F, Staal FJ, Plum J and Leclercq G: Homeobox gene expression profile in human hematopoietic multipotent stem cells and T-cell progenitors: implications for human T-cell development. Leukemia 17: 1157-1163, 2003

28. Garin E, Lemieux M, Coulombe Y, Robinson GW and Jeannotte L: Stromal Hoxa5 function controls the growth and differentiation of mammary alveolar epithelium. Dev Dyn 235: 1858-1871, 2006.

29. Lu Z, Hardt J and Kim JJ: Global analysis of genes regulated by HOXA10 in decidualization reveals a role in cell proliferation. Mol Hum Reprod 14: 357-366, 2008.

30. Kongsuwan K, Webb E, Housiaux P and Adams JM: Expression of multiple homeobox genes within diverse mammalian haemopoietic lineages. EMBO J 7: 2131-2138, 1988.

31. Christensen KL, Patrick AN, McCoy EL and Ford HL: The six family of homeobox genes in development and cancer. Adv Cancer Res 101: 93-126, 2008.

32. Cillo C, Cantile M, Faiella A and Boncinelli E: Homeobox genes in normal and malignant cells. J Cell Physiol 188: 161-169, 2001.

33. Care A, Felicetti F, Meccia E, Bottero L, Parenza M, Stoppacciaro A, Peschle C and Colombo MP: HOXB7: a key factor for tumor-associated angiogenic switch. Cancer Res 61: 6532-6539, 2001.

34. Wu X, Chen H, Parker B, Rubin E, Zhu T, Lee JS, Argani P and Sukumar S: HOXB7, a homeodomain protein, is overexpressed in breast cancer and confers epithelial-mesenchymal transition. Cancer Res 66: 9527-9534, 2006.

35. Srebrow A, Friedmann Y, Ravanpay A, Daniel CW and Bissell MJ: Expression of Hoxa-1 and Hoxb-7 is regulated by extracellular matrix-dependent signals in mammary epithelial cells. J Cell Biochem 69: 377-391, 1998. 